\title{
Best Practices for Community-Engaged Research with Pacific Islander Communities in the US and USAPI: A Scoping Review
}

\author{
Pearl A. McElfish, PhD, MBA, Karen Yeary, PhD, Ka'imi A. Sinclair, PhD, MPH, Susan \\ Steelman, MLIS, Monica K. Esquivel, PhD, RDN, Nia Aitaoto, PhD, MS, MPH, Keawe'aimoku \\ Kaholokula, PhD, Rachel S. Purvis, PhD, Britni L. Ayers, PhD \\ PEARL A. MCELFISH, KAREN YEARY, SUSAN STEELMAN, RACHEL S. PURVIS, and BRITNI \\ L. AYERS are affiliated with University of Arkansas for Medical Sciences. KA'IMI A. SINCLAIR is \\ affiliated with Washington State University. MONICA K. ESQUIVEL and KEAWE'AIMOKU \\ KAHOLOKULA are affiliated with University of Hawaii Manoa. NIA AITAOTO is affiliated with \\ University of Utah.
}

\begin{abstract}
This scoping review identifies the best practices of community-based participatory research with Pacific Islanders in the United States and United States Affiliated Pacific Islands. Eighty-four articles from January 2000 to December 2017 were included in the review. Best practices included the importance of engaging Pacific Islander community leaders as research staff, community coinvestigators, and community advisory board members. Best practices also focused on removing barriers to research by using participants' native languages, conducting research within the geographic community, and spending significant time to build trust. Novel best practices included honoring Pacific Islanders' cultural practices such as protocols for engagement, reciprocity, and social and spiritual inclusiveness and honoring Pacific Islanders' collectivist cultural structure. The goal of this scoping review is to aid community-academic partnerships working to improve the health of Pacific Islanders.
\end{abstract}

\section{Keywords}

Pacific Islander; scoping review; community-based participatory research; health disparities

Pacific Islanders are the second fastest growing population in the United States (U.S.), increasing $40 \%$ between 2000 and 2010. ${ }^{1-3}$ More than a million Pacific Islanders live in the U.S., and by 2030 the U.S. Census Bureau projects that the Pacific Islander population will reach two million. ${ }^{4}$ Pacific Islanders are indigenous to the Pacific regions of Polynesia (e.g., Hawai'i, Tonga, Sāmoa,), Melanesia (e.g., Fiji and Vanuatu), and Micronesia (e.g., Marshall Islands, Federated States of Micronesia, Guam, and Palau). ${ }^{5}$ The U.S. Office of Management and Budget has aggregated Pacific Islanders under a single racial/ethnic category called, Native Hawaiians and Other Pacific Islanders (referred to collectively as

Please address all correspondence to Pearl A. McElfish, University of Arkansas for Medical Sciences, Northwest Campus, 1125 N. College Ave, Fayetteville, AR 72701.pamcelfish@uams.edu. Conflict of Interest: None. 
Pacific Islanders in this review). While most Pacific Islanders live in the U.S. Affiliated Pacific Islands (USAPI), Hawai'i, and California, the fastest growth is taking place in middle America in states such as Arkansas, Kansas, Nevada, and Oklahoma. ${ }^{1-3}$

Historically, Pacific Islanders have been aggregated with Asian Americans in many population-based studies in the U.S.; this data aggregation has masked disparities between these two heterogenic populations. ${ }^{6-10}$ However, existing research indicates that Pacific Islanders suffer from profound health disparities compared with the general U.S. population. 11,12 Pacific Islanders bear a disproportionate burden of diabetes, cardiovascular disease, obesity, and cancer. ${ }^{13-17}$ While national data on life expectancy for Pacific Islanders is not available due to aggregation, state and U.S. territory data document that the life expectancy of Pacific Islanders is 71 years, lower than the national average of 79 years. ${ }^{18,19}$

Pacific Islanders' health disadvantages can be attributed to many factors, including a history marked by trauma, exploitation, and exclusion. ${ }^{20-23}$ Historical trauma has caused deep apprehension and distrust of Western medicine and research, ${ }^{24,25}$ which heightens the ethical challenges that must be addressed in order to conduct research with Pacific Islanders. 24,26 One way to address historical trauma in Pacific Islander communities and their exclusion from health research is through community-based participatory research (CBPR), 24,26 an approach to research that equitably involves community members/organizations in research (e.g., through sharing of power and resources). ${ }^{27,28}$

The literature on best practices for engaging racial/ethnic minority participation in CBPR has grown exponentially over the past decade. ${ }^{29-50}$ While numerous reviews have reported CBPR best practices, ${ }^{29-47}$ these reviews have rarely included research with Pacific Islander communities. This lack of research representation leaves a critical gap in the literature for partners who seek to understand CBPR practices unique to Pacific Islanders for the purpose of addressing the profound health disparities in this rapidly growing population. Thus, this article presents a scoping review of published studies that have implemented CBPR with Pacific Islander communities in the U.S. and USAPI. The USAPI includes three Territories (American Samoa, Guam, and the Commonwealth of the Northern Mariana Islands) and three Freely Associated States (Federated States of Micronesia, Republic of the Marshall Islands, and the Republic of Belau [also known as Palau]).

\section{Methods}

We conducted a scoping review in conformance with modified items of the Preferred Reporting Items for Systematic Reviews and Meta-Analysis (PRISMA). ${ }^{51,52}$ The review sought to answer the following question: What are the best practices authors identify for conducting CBPR with Pacific Islanders in the U.S. and USAPI?

\section{Eligibility criteria.}

Articles were selected according to criteria detailed in Box 1.

Articles published between January of 2000 and December of 2017 in peer-reviewed journals were selected for this review. Articles were limited to research conducted with 
participants residing in the U.S. and USAPI. Articles that included Asian Americans aggregated with Pacific Islanders were not included. Articles that discussed the use of CBPR or related engagement approaches were included. For the purposes of establishing article eligibility, CBPR was defined as a partnership approach to research that equitably involves community stakeholders in the research process. ${ }^{28,53-55}$

\section{Databases.}

The main literature search for this scoping review was conducted in November of 2017 with auto alerts subsequently following the literature until March 2018. Biomedical databases were searched for articles meeting the eligibility criteria and focused on English language items with January 2000-December 2017 publication dates. These databases included: MEDLINE (OVID), MEDLINE In Process \& Daily Updates (OVID), International Pharmaceutical Abstracts (OVID), Science Citation Index, and Social Sciences Citation Index (both via Web of Science). The Cumulative Index of Nursing and Allied Health Literature, PsycINFO, SocINDEX, and Healthy Policy Reference Center were all searched through the EBSCO platform. The All EBM Reviews collection (OVID) included: American College of Physicians Journal Club, Cochrane Database of Scoping Reviews, Databases of Abstracts of Reviews of Effects, Cochrane Central Register of Controlled Trials, Cochrane Methodology Register, Health Technology Assessment, and the National Health Service Economic Evaluation Database. The World Health Organization's Global Index Medicus database was also searched for international items.

\section{Search strategy.}

The search strategy was developed by a medical librarian in consultation with the authors. MEDLINE searches combined Medical Subject Headings with advanced text word search techniques that included truncation, adjacency searching, and extensive strings for Pacific Islander populations. Similar search methodologies were implemented in databases on other platforms. All search strategies were documented. A full discussion of terms is provided in the published protocol. ${ }^{56}$

\section{Data management.}

Search results were exported, stored, and shared using RefWorks (version 2.0), ${ }^{21}$ an Internet-based reference management service. Duplicate records were removed from search results by using RefWorks' duplicate-check function with manual oversight prior to duplicate removal. De-duplicated records were exported from RefWorks into a Microsoft Excel 2013 (version 15.0) spreadsheet to facilitate the article selection process. ${ }^{22}$

\section{Article selection process.}

The title and abstract of each article were reviewed to determine whether the article met all the eligibility criteria. If the title and abstract did not provide enough information to confirm eligibility, the full article was reviewed by two researchers to determine if it met the inclusion criteria. If it was still unclear if the article was eligible, a third researcher reviewed the article and a final decision was made based on consensus. When tabulating quantitative results, care was taken to avoid double-counting single articles. 


\section{Data extraction process.}

Data extraction from each eligible article was performed by two researchers independently.

Researchers compared the results of the extraction process and discrepancies in extracted data were discussed and resolved between the researchers. If there were multiple publications of a single eligible article, data were extracted from multiple publications. Before data extraction began, researchers piloted the extraction spreadsheet on a small sample of 20 eligible articles and adjusted the extraction spreadsheet or extraction procedures as needed.

\section{Data items.}

The following data were extracted from each eligible article. (Box 2)

\section{Data synthesis.}

Researchers trained in qualitative methods coded the eligible articles using an inductive coding process to generate a list of emerging best practices. Emergent themes were organized into a code book that was used to classify each article. After all articles were coded, the data was synthesized into a summary of emergent themes. This process allowed the researchers to create salient summaries of best practices. ${ }^{57,58}$ Best practices are provided in synthesized summaries of emergent themes. In addition, frequency and percentages were calculated for each emergent theme.

\section{Results}

The initial searches across listed databases were run November 21, 2017. Auto alerts for the main strategy were included through March 2018 when data analysis began. In total, 601 articles were retrieved from the literature searches. Total duplicate articles excluded were 241. The remaining 360 articles were reviewed for relevance to the research question and four abstracts were excluded. The remaining 356 full-text articles were reviewed and 273 articles were excluded for not meeting the inclusion criteria (e.g., aggregated data, not CBPR, not in U.S. or USAPI); thus, we included 83 articles in the review as detailed in Figure 1.

The articles presented in this scoping review published best practices for CBPR with Pacific Islander communities in the U.S. and USAPI. Of the 83 articles included in the scoping review, 33\% used qualitative methods such as focus groups and/or individual interviews, $33 \%$ of the articles used mixed methods or combined methods, $18 \%$ of the articles used quantitative methods that statistically analyzed data collected, and $17 \%$ of the articles were descriptive articles of the CBPR process (Table 1).

Thirty-five percent of the studies were conducted with multiple Pacific Islander subgroups, $35 \%$ of the articles were exclusively conducted with Native Hawaiians, followed by $16 \%$ with Marshallese, $7 \%$ with Samoan, 2\% with Tongan, $2 \%$ with Chuukese, $1 \%$ with Chamorro, and $1 \%$ with Yapese. (Table 2) The studies were primarily conducted in Hawai' $i$ (41\%), followed by the continental U.S. (34\%), multiple locations in the U.S. and USAPI (3\%), and exclusively in the USAPI (6\%) (Table 3). 
Many of the studies conducted used both male and female participants (51\%), whereas (19\%) focused solely on women, and (5\%) of studies focused solely on men. Twenty-five percent of studies did not present data by sex. The majority of the studies included adults over the age of $18(67 \%), 6 \%$ of studies were conducted with children, and $6 \%$ were conducted with adults and children (Table 4). Twenty percent of the articles did not specify participants' ages. Cancer studies (19\%) dominated the literature, followed by diabetes $(18 \%)$, non-specific health research (16\%), obesity (14\%), drug prevention (8\%), intimate partner violence (5\%), cardiovascular disease (5\%), maternal health (4\%), sexually transmitted disease (4\%), biospecimen research (2\%), genetic studies (2\%), and depression (1\%) (Table 5).

\section{Qualitative results.}

Four themes with salient sub-themes emerged from the review: 1) Engaging community leaders, 2) Facilitators and barriers to research, 3) Honoring cultural practices, and 4) Honoring collectivist cultural structure (see Table 6).

\section{Engaging community leaders.}

Consistent with the extant CBPR literature, ${ }^{59-61}$ the most commonly articulated best practices were the importance of fully partnering with community leaders in the research process and the research being co-led by the community partners. Most reported multiple ways to engage community leaders. Within the theme Engaging community leaders, there were four subthemes: 1) Pacific Islander research staff (81\%), 2) Community-based organizations (80\%), 3) Community advisory board (54\%), and 4) Faith-based leaders/Faithbased organizations or elders (22\%).

Pacific Islander research staff.-The majority (81\%) of articles in the review discussed the importance of Pacific Islander staff serving in formal research and leadership positions for the study's success. ${ }^{62-123}$ Pacific Islander research staff served as gatekeepers, advocates, and health educators in their communities. The reviewed articles described the presence of Pacific Islander research staff as a way to build trust within the community and ensure that research is in congruence with cultural practices. Pacific Islander research staff were able to make connections within the community, which facilitated the recruitment of participants. Some articles discussed how the presence of Pacific Islander research staff also provided an opportunity for increased candor and reduced participants' fear of being misunderstood. ${ }^{85,106}$ Most importantly, by engaging Pacific Islander research staff, the broader Pacific Islander community gained a sense of empowerment because Pacific Islanders shaped decisions about the conduct of the study, had access to all study information, and provided input in the use of the study results.

Community-based organizations.-The involvement of community leaders through community-based organizations was discussed in $80 \%$ of the articles. 62-67,69,70,72-95,98-112,116,117,120-136 The involvement of community-based organizations was cited as a way to leverage, build, and sustain leadership within the Pacific Islander community. ${ }^{81}$ Community-based organizations (e.g., federally qualified community health centers and grassroots non-profit community-based organizations) were described as playing 
a direct role in the research design, study conduct, and dissemination of the results back to the community. Community-based organizations were described as particularly important to the recruitment of participants. Furthermore, community-based organizations often served as the locations for study activities. Conducting study activities at the community-based organization was discussed as a way to ensure that research activities were more easily accessible by removing both geographic and cultural barriers.

Community advisory board.-More than half (54\%) of the reviewed articles mentioned partnering with a community advisory board. $62,64-67,69,70,72-76,78-84,85-95,98-112,116,117,120-124,125-138$ Specifically, community advisory boards were discussed as contributing to the overall success of research projects by engaging multiple members across a broad spectrum of the community rather than from only one community-based organization or segment of the community. Engagement of a community advisory board was cited as a way to ensure the needs and desires of the wider community were included in the research. ${ }^{72,109}$

Faith-based leaders and community elders.-The engagement of faith-based leaders and community elders as a best practice was identified in $22 \%$ of the articles, $62-64,77,84,85,93,95,97,101,102,108,110,118,119,126,128,133,135,137-138$ which is consistent with

CBPR best practices already identified concerning some populations (e.g., rural African Americans). ${ }^{139,140}$ When discussing the involvement of faith-based leaders, articles cited the role of faith-based leaders as extending beyond religious beliefs and discussed the role of faith-based organizations and faith-based leaders as representing the clan structures, and faith-based leaders as having a role almost similar to that of a governmental leader within the community. ${ }^{101,128}$ Broadly, the engagement of faith-based leaders and community elders was cited as an imperative step in attaining access to the community, and was discussed as an important part of recruitment and retention of participants. Most importantly, the reviewed articles emphasized the importance of engaging faith-based leaders and community elders to ensure practices were culturally appropriate.

\section{Facilitators and barriers to research.}

The reviewed articles focused on best practices for overcoming common barriers to stakeholders engaging in research and identified facilitators. The best practices identified were consistent with other CBPR studies in other populations and included conducting research in the primary or native language of participants, holding meetings in a convenient community location rather than at the university or research institute, and giving appropriate time to develop relationships within the community. The subthemes identified were: 1) Community location (42\%), 2) In-language (36\%), and 3) Time to develop relationships $(23 \%)$.

Community location.-Almost half (42\%) of the reviewed articles described the need to hold research meetings in an easily accessible community location. $62,68,75,76,78,84,86,87,90,92,93,95,97,99-102,105,107-109,112,113,118,119,120,124,125,128,141-142$

Transportation was consistently discussed as a barrier to conducting research with Pacific Islander communities. For example, Tanjasiri et al. described that among Marshallese 
communities, transportation is a common barrier and that community staff typically need to take them to and from the assessment site. ${ }^{102}$ The location was important in overcoming transportation barriers, and was also important in creating a comfortable environment for stakeholders and participants. Common locations included community-based organization and faith-based organization buildings.

In-language.-More than one-third (36\%) of the articles reviewed discussed the necessity of having research studies conducted in the native language of the Pacific Islander community. ${ }^{62,63,66,71,72,75-79,84,86-89,91-95,98,100,101,103,104,108,110,111,115,119 ~ B e s t ~ p r a c t i c e s ~}$ included providing both written and verbal communication in native language. One notable exception to language translation was research with Native Hawaiian community members because most Native Hawaiians are fluent in English, and many Native Hawaiians do not speak Hawaiian. ${ }^{143}$ However, some articles demonstrated the inclusion of the Hawaiian language alongside Native Hawaiian imagery in an attempt to revive cultural traditions among the participants and increase salience. ${ }^{66,75,87}$ Overcoming language barriers included more than simply translating information into the native language of participants; best practices focused on ensuring the appropriate literacy level and ensuring culturallyappropriate language was taken into consideration. Articles documented that it is important to consider the formality of the information being provided and to ensure culturally appropriate examples in verbal and written communication. ${ }^{71,72,63}$

Time to develop relationships.-The reviewed articles described Pacific Islanders' priority for relationships and described how time spent in relationships was foundational for the conduct of research. Twenty-three percent of the articles explicitly discussed the need to invest significant amounts of time into building and nurturing meaningful relationships between community members and researchers, $76,78,81,85,86,101,106-108,111,112,115,125,128,129,131,132,138$ and many other articles that did not cite this concept directly alluded to the importance of building relationships. Furthermore, the time spent building relationships was described as important because research could not take place until researchers increased their knowledge of the community's culture and established trust within the community. For example, in the Native Hawaiian culture there is a preference for the process of building a social connection or linkage referred to as pilina (to engage in a relationship). ${ }^{112}$ This process can require substantial time; Chung-Do et al. discussed that communities prefer working with researchers who are vested in a long-term relationship that extends beyond the research study timetable as this is more conducive to true collaborative partnerships. ${ }^{107}$ Articles described the importance of taking the time to approach multiple community groups to gain a more comprehensive understanding of health needs and possible solutions. ${ }^{106}$ Although this approach required more time and resources, it was important because it allowed community members to be more invested in the research, time to develop trust, and the opportunity to assess the intentions of the researchers.

\section{Honoring cultural practices.}

Honoring the practices of Pacific Islander cultures was discussed as a critical component of research with this community. Almost half (44\%) of the articles discussed adapting existing evidence-based interventions to ensure they were appropriate to the specific Pacific Islander 
culture of the research study. The sub-themes for honoring cultural practices includes: 1) Incorporating faith beliefs (23\%), 2) Engaging in talk story (19\%), 3) Sharing meals during the conduct of research (17\%), and 4) Separating activities by sex (7\%).

Incorporating faith beliefs.-Incorporating faith, which primarily include Christian and traditional beliefs, was described in $23 \%$ of the articles. $62-64,67,75,78,84,85,95,101,102,104,108,110,119,128,131,134$ Many of the reviewed articles discussed the importance of incorporating faith into research studies, either by ensuring that the research protocol reflected specific faith-based practices or conducting studies within a church setting. Faith was discussed as a particularly important component in many Pacific Islander subgroups. For example, Leslie et al. described the importance of beginning all focus groups with a pule (prayer) for Native Hawaiian gatherings not only to bless the food but to solicit spiritual guidance for a productive and positive discussion. ${ }^{85}$ Panapasa et al. described the importance of incorporating faith into CBPR research with Pacific Islanders because coupling faith with health provided a vehicle for a collective healthy focus within the community. ${ }^{128}$

Oral traditions and talk story.-Engaging oral traditions and talk story was another cultural practice that emerged as important across multiple Pacific Islander communities $(19 \%) .66,67,72,74,75,77,94,95,99,104,108,112,126,131$ The term talk story was used to describe a relaxed conversation involving a reciprocal, symbiotic exchange of thoughts, beliefs, and feelings often told in a story format. ${ }^{72}$ The use of talk story or other relational oral communication was described as an important component of CBPR among Pacific Islander communities because it represented a shift in the power from the researchers asking for information from the community to a more fluid exchange where multiple parties shared equally and time was spent to build trust. ${ }^{99}$ Some articles discussed the use of focus group methods as leveraging Pacific Islander oral traditions and being similar to talk story and therefore increasing the effectiveness of focus groups within Pacific Islander communities. 107,111 Other studies used talk story to build rapport because it demonstrated respect for Pacific culture and customs for dialogue with Pacific Islander communities. ${ }^{99}$

Sharing a meal.-Another cultural practice described in $17 \%$ of the articles was the sharing of a meal. ${ }^{64,67,70,73,75,77,80,85,97,104,112,118,131,135,138}$ For many Pacific Islander communities, food is the center of social interaction and is described as a vital part of gatherings. Communal meals facilitated the opportunity for researchers and participants to engage with one another as equals and potentially as extensions of family and community. Sharing meals was also described as an opportunity to share cultural practices and promote healthy foods.

Separation of participants by sex.-Lastly, while true of only $7 \%$ of the overall articles reviewed, some articles discussed the importance of separating participants based on sex for research activities including biometric measurements and/or the discussion of sensitive topics. ${ }^{62,64,72,75,101,108}$ Furthermore, articles often described the importance of the research staff member collecting the biometric or sensitive survey data to be the same sex as the participant. 


\section{Honoring collectivist cultural structure.}

Pacific Islander culture is highly collectivist and values the needs of the group (family and/or community) over the individual. This theme detailed CBPR best practices unique to Pacific Islanders and included three subthemes: 1) Group engagement (55\%), 2) Family engagement (39\%), and 3) Community consent (24\%).

Group engagement.-Over half (55\%) of the articles discussed the importance of implementing research in group settings citing using focus group discussions and collecting data via surveys in groups as examples. 62-64,66,67,70-72,74,76-81,85-88,90,92,93,95-97,99,100,102,104,106,108,109,112,113,116,119,121,124,125,127,134,144,145

Group engagement in study activities supported increased community and participant comfort with research activities and was described as reflective of Pacific Islander cultural values, yielding enhanced involvement from Pacific Islander participants. For example, Kagawa-Singer et al. documented that Pacific Islander participants may be more comfortable providing information if friends from their community were allowed to participate as well. ${ }^{78}$

Family engagement.-More than a third (39\%) of the articles reviewed discussed the importance of focusing on the family unit when conducting research and/or incorporating family into the intervention design. 62,63,66,68,70-72,75-79,88,90,96-101,104,109,110,112,113,118,119,121,125,127,134,145 The definition of family cited in some articles went beyond parents, children, and siblings to include extended family members such as aunts, uncles, and cousins. The definitions of family also extended beyond biology and included close friends or people from the same clan or island. Some Pacific Islander communities, such as the Marshallese, were cited as articulating that individual health changes do not occur independent of the family. ${ }^{90}$

Community consent.-In addition to group and family engagement, $24 \%$ of the articles discussed the importance of obtaining community consent for research studies. 62,66,67,72,74,78,82,83,85,89-93,100,101,107,108,128 When seeking broader community consent, Pacific Islander community elders and faith-based leaders were often pointed to as those needing to provide approval or endorsement to conduct research within Pacific Islander communities. Further, in many Pacific Islander communities, such as Native Hawaiian, articles mentioned the importance of presenting a proposed research project orally to the community and obtaining approval from the community in general prior to starting the study. 100

\section{Discussion}

The review of the literature between January 2000 and December 2017 revealed several best practices for conducting CBPR in Pacific Islander communities in the U.S. and USAPI. One of the primary best practices was the importance of engaging community leaders through multiple strategies (e.g., as research staff, as community co-investigators, and/or as community advisory board members), which is consistent with the CPBR literature in other populations. ${ }^{28,49,146}$ Prior reviews in other populations have demonstrated the importance of 
engaging research staff that reflect the community being engaged in research on their racial/ ethnic and socioeconomic characteristics. Furthermore, the findings are consistent with other studies that have documented the importance of community-based organizations, community advisory boards, faith-based leaders/faith-based organizations, and community elders in ensuring a successful CBPR project. ${ }^{48}$ The meaningful inclusion of community members in the decision-making process and in the implementation of a study helps to build community capacity to engage in health promotion activities, to provide cultural relevance and context, and to increase the likelihood that the findings or products of the research will be used and sustained beyond the life of the research project. ${ }^{147}$

Also consistent with prior CBPR literature is the best practice of removing barriers to research by using the participants' native language and cultural perspectives, holding meetings in an easily accessible location for community partners, and spending significant time in the community to build trust and to understand their concerns, values, customs, and preferred ways of addressing issues. This finding is consistent with CBPR literature that has included other Indigenous populations. ${ }^{148-154}$ This study extends the current literature because Pacific Islander communities have not been included in prior CBPR reviews. ${ }^{48}$ In addition to confirming what other CBPR reviews have documented, this study revealed several best practices unique to Pacific Islanders to consider when conducting CBPR with this population. These novel findings focused on best practices for honoring cultural practices and collectivist cultural structure.

Honoring the cultural practices, such as protocols for engagement, reciprocity, and social and spiritual inclusiveness was found to be important in many of the reviewed articles. Specifically, sharing meals, use of faith-based approaches, and research methods that respected oral traditions and preference for talk story style of communication, and separate research engagement based upon the sex of the participants emerged as important factors for CBPR work with Pacific Islanders. These cultural practices were consistently reported across Pacific Islander subgroups and have significant implications for the conduct of CBPR. The use of relational oral communication and talk story in the Pacific Islander community may make qualitative methods, including focus groups, particularly effective methods of research with Pacific Islanders. Some of the CBPR practices may be difficult to implement. For example, federal grant funding and universities' policies often restrict the expenditures for food, ${ }^{148}$ thus additional funding or additional flexibility in funding may be needed to engage Pacific Islanders. Separating engagement of participants by sex and matching the sex of the data collector to the sex of the participant is also important, but may be difficult for some studies with limited budgets. Most of the practices are not as common in the broader CBPR literature, but some of the best practices have been documented when engaging in CBPR with Native American communities. ${ }^{149-154}$

Honoring the collectivistic cultural structure of Pacific Islanders also emerged as a CBPR best practice. Honoring the collectivist culture entailed conducting research within the collective group or family, which increased participation while conveying respect for cultural practices. Reviewed studies reported that working within Pacific Islanders' collectivistic cultural structure is often counter to more individualistic cultures (e.g., Western) and thus may require a greater degree of flexibility on the part of the investigators with an 
individualistic cultural framework. For example, informed consent is typically elicited individually, and most Western institutional review boards may not favor group consent, which may influence the individual participant consent. Future work should explore how to translate research practices from an individualistic framework to a collectivist one. Furthermore, when considering how to engage families in health behavior change or research, researchers would benefit from considering the Pacific Islander definition of family, which often focuses on the extended (e.g., cousins and aunts) and non-biological relations. Many of these CBPR best practices are not as common in the broader CBPR literature; however, they are similar to best practices for CBPR with Native American communities. ${ }^{155-157}$

Cancer, diabetes, and obesity were the dominant health disparities addressed in the reviewed articles. This is not surprising given that these medical conditions are highly prevalent in and disproportionally burdensome (e.g., more likely to be diagnosed at latter stages of cancer) for Pacific Islander communities. ${ }^{13,152}$ However, the limited research in other health areas, including mental and sexual health, may signify areas that should be explored within the Pacific Islander community. Specifically, mental and behavioral health concerns that include substance use and abuse, have been identified as high-priority areas for Pacific Islander communities. ${ }^{153}$

\section{Limitations.}

It is important to note that this review did not attempt to evaluate the quality of the community engagement or the research methods. Although Pacific Islanders are similar in some regards, they are not a homogeneous group; thus, this review was limited in the ability to assess best practices that may be distinct to specific subgroups of Pacific Islanders. A third of the articles did not disaggregate Pacific Islanders subgroups, thus complicating assessment of best practices by subgroup. Despite these limitations, this scoping review provided new information about CBPR best practices unique to Pacific Islanders that can be considered in future participatory work. Future studies should explore shared and distinct practices among various Pacific Islander subgroups.

\section{Conclusion.}

Using a CBPR approach in collaborating with Pacific Islanders offers the promise of mitigating the effects of historical trauma, ensuring cultural safety and relevance, and achieving health equity in these communities. Such approaches have already succeeded in recruiting and retaining participants from underserved populations and in attaining significant intervention effects. ${ }^{48,49}$ Some researchers may find it difficult to implement the best practices identified through this literature review. As is common in CBPR, a great investment of time, energy, and patience is needed to build equitable partnerships for the purpose of eliminating health disparities. Yet the investment is not without its rewards, which can include research engagement, building community and academic capacity, sustainability, and health equity. Each community has its own set of unique practices that both academic and community researchers should identify and implement. The hope of this scoping review is to expedite this often long-term process for other community-academic partnerships working to improve the health of Pacific Islanders. 


\section{Acknowledgments}

Financial Support: The CBPR partnership support was provided from the University of Arkansas for Medical Sciences Translational Research Institute (grant UL1TR000039), which was funded through the NIH National Center for Research Resources and National Center for Advancing Translational Sciences. Research reported in this publication was also partially supported by the National Institute of General Medical Sciences of the National Institutes of Health under Award Number P20GM109096. The content is solely the responsibility of the authors and does not necessarily represent the official views of the National Institutes of Health.

\section{References}

1. Grieco E. The Native Hawaiian and other Pacific Islander population: 2000. Washington, DC: United States Census Bureau, 2001 Available at: http://www.census.gov/prod/2001pubs/ c2kbr01-14.pdf.

2. United States Census Bureau. 2010 Census shows more than Half of Native Hawaiians and Other Pacific Islanders report multiple races. Washington, DC: United States Census Bureau, 2012.

3. Hixson L, Hepler B, Kim MO. The Native Hawaiian and Other Pacific Islander Population: 2010. Washington, DC: United States Census Bureau, 2012 Available at: http://www.census.gov/prod/ cen2010/briefs/c2010br-12.pdf.

4. Asian Americans Advancing Justice. A community of contrasts: Asian Americans, Native Hawaiians and Pacific Islanders in the Midwest. Los Angeles, CA: Asian American Center for Advancing Justice, 2012.

5. Fischer SR. A history of the Pacific Islands. New York, NY: Palgrave; 2002.

6. Applied Research Center, National Council of Asian Pacific Americans. Best Practices: Researching Asian Americans, Native Hawaiians and Pacific Islanders. Washington, DC: National Council of Asian Pacific Americans, 2017 Available at: https://d3n8a8pro7vhmx.cloudfront.net/ncapa/ pages/33/attachments/original/1430842908/Best-Practices-AANHPI.pdf?1430842908.

7. Ro M, Lee AK. Out of the Shadows: Asian Americans, Native Hawaiians, and Pacific Islanders. Am J Public Health. 2010 5;100(5):776-8. Epub 2010 Mar 18. 10.2105/AJPH.2010.192229 [PubMed: 20299635]

8. Srinivasan S. Toward improved health: disaggregating Asian American and Native Hawaiian Pacific Islander data. Am J Public Health. 2000 11;90(11):1731-4. 10.2105/AJPH.90.11.1731 [PubMed: 11076241]

9. Nguyen A, Chawla N, Noone A, et al. Disaggregated Data and Beyond: future queries in cancer control research. Cancer Epidemiol Biomarkers Prev. 2014 11;23(11):2266-72.

10.1158/1055-9965.EPI-14-0387 [PubMed: 25368401]

10. Ahmad FZ, Weller CE. Reading between the data: the incomplete story of Asian Americans, Native Hawaiians, and Pacific Islanders. Washington, DC: Center for American Progress, 2014 Available at: https://www.americanprogress.org/wp-content/uploads/2014/03/AAPI-report.pdf.

11. Mau M. Health and health care of Native Hawaiian \& Other Pacific Islander older adults. In: Periyakoil VS, eds. Stanford, CA: Stanford School of Medicine, 2010 Available at: https:// geriatrics.stanford.edu/ethnomed/hawaiian_pacific_islander.html.

12. Moy KL, Sallis JF, David KJ. Health indicators of Native Hawaiian and Pacific Islanders in the United States. J Community Health. 2010 2;35(1):81-92. 10.1007/s10900-009-9194-0 [PubMed: 19856087]

13. Tung WC. Diabetes among Native Hawaiians and Pacific Islanders. Home Health Care Management \& Practice. 2012;24(6):309-11. 10.1177/1084822312454002

14. Mau MK, Sinclair K, Saito EP, et al. Cardiometabolic health disparities in native Hawaiians and other Pacific Islanders. Epidemiol Rev. 2009;31:113-29. Epub 2009 Jun 16. 10.1093/ajerev/ mxp004 [PubMed: 19531765]

15. Okihiro M, Harrigan R. An overview of obesity and diabetes in the diverse populations of the Pacific. Ethn Dis. 2005 Autumn;15(4 Suppl 5):S5-71-80.

16. Buenconsejo-Lum L, Navasca D, Jeong Y, et al. Cancer in the U.S. Affiliated Pacific Islands 20072011. Honolulu, HI: Pacific Regional Central Cancer Registry, 2014 Available at: http:// 
pacificcancer.org/pacp-resources/key-cancer-publications/

PIJ_Cancer_FactsandFigures_FINAL_031514.pdf.

17. Office of Minority Health. Profile: Native Hawaiian and Pacific Islanders. Washington, DC: Health and Human Services, 2015 Available at: http://minorityhealth.hhs.gov/omh/browse.aspx? $1 \mathrm{vl}=3 \& l v l i d=65$.

18. Arias E. United States life tables, 2001. Natl Vital Stat Rep. 20042 18;52(14):1-38.

19. Secretariat of the Pacific Community (SPC). Republic of the Marshall Islands 2011 census report. Noumea, New Caledonia: SPC, 2011 http://prism.spc.int/images/census_reports/ Marshall_Islands_Census_2011-Full.pdf.

20. Cooke S. In mortal hands: a cautionary history of the Nuclear Age. New York, NY: Bloomsbury USA, 2010.

21. Pollock NJ. Health transitions, fast and nasty: exposure to nuclear radiation. Pacific Health Dialog. 2002 9;9(2):275-82. [PubMed: 14736115]

22. Guyer RL. Radioactivity and rights: clashes at Bikini Atoll. Am J Public Health. 2001 9;91(9):1371-6. [PubMed: 11527760]

23. Barker H. Bravo for the Marshallese: regaining control in a post-nuclear, post-colonial world. Belmont, CA: Wadsworth, 2012.

24. Minkler M. Ethical challenges for the "outside" researcher in community-based participatory research. Health Educ Behav. 2004 12;31(6):684-97. [PubMed: 15539542]

25. Evans-Campbell T. Historical trauma in American Indian/Native Alaska communities: a multilevel framework for exploring impacts on individuals, families, and communities. J Interpers Violence. 2008 3;23(3):316-38. [PubMed: 18245571]

26. Wallerstein N. Power between evaluator and community: Research relationships within New Mexico's healthier communities. Soc Sci Med. 1999 Jul;49(1):39-53.

27. Wallerstein N, Duran B. Using community-based participatory research to address health disparities. Health Promot Pract. 2006 7;7(3):312-23. Epub 2006 Jun 7. 10.1177/1524839906289376 [PubMed: 16760238]

28. Minkler M, Wallerstein N. (eds.). Community-based participartory research for health: from process to outcomes. San Francisco, CA: Jossey-Bass, 2008.

29. Nwanyanwu KH, Grossetta Nardini HK, Shaughness G, et al. Systematic review of communityengaged research in ophthalmology. Expert Rev Ophthalmol. 2017;12(3): 233-41. Epub 2017 Apr 19. 10.1080/17469899.2017.1311787 [PubMed: 29333193]

30. Commodore A, Wilson S, Muhammad O, et al. Community-based participatory research for the study of air pollution: a review of motivations, approaches, and outcomes. Environ Monit Assess. 2017 8;189(8):378 Epub 2017 Jul 8. 10.1007/s10661-017-6063-7 [PubMed: 28685368]

31. Tremblay MC, Martin DH, Macaulay AC, et al. Can we build on social movement theories to develop and improve community-based participatory research? A framework synthesis review. Am J Community Psychol. 2017 6;59(3-4):333-62. Epub 2017 May 4. 10.1007/s10661-017-6063-7 [PubMed: 28471507]

32. Vaughn LM, Jacquez F, Lindquist-Grantz R, et al. Immigrants as research partners: a review of immigrants in community-based participatory research (CBPR). J Immigr Minor Health. 2017 12;19(6):1457-68. 10.1007/s10903-016-0474-3 [PubMed: 27491305]

33. Souleymanov R, Kuzmanovi D, Marshall Z, et al. The ethics of community-based research with people who use drugs: results of a scoping review. BMC Med Ethics. 20164 29;17(1):25 10.1186/ s12910-016-0108-2 [PubMed: 27129927]

34. Coughlin SS, Smith SA. Community-based participatory research to promote healthy diet and nutrition and prevent and control obesity among African-Americans: a literature review. J Racial Ethn Health Disparities. 2017 4;4(2):259-68. Epub 2016 Apr 8. 10.1007/s40615-016-0225-0 [PubMed: 27059053]

35. Sofolahan-Oladeinde Y, Mullins CD, Baquet CR. Using community-based participatory research in patient-centered outcomes research to address health disparities in under-represented communities. J Comp Eff Res. 2015 9;4(5):515-23. Epub 2015 Oct 5. 10.2217/cer.15.31 [PubMed: 26436953]

36. Mikesell L, Bromley E, Khodyakov D. Ethical community-engaged research: a literature review. Am J Public Health. 2013 12;103(12):e7-14. Epub 2013 Oct 17. 10.2105/AJPH.2013.301605 
37. Vaughn LM, Wagner E, Jacquez F. A review of community-based participatory research in child health. MCN Am J Matern Child Nurs. 2013 Jan-Feb;38(1):48-53. 10.1097/ NMC.0b013e31826591a3 [PubMed: 23232779]

38. Salimi Y, Shahandeh K, Malekafzali H, et al. Is community-based participatory research (CBPR) useful? A systematic review on papers in a decade. Int J Prev Med. 2012 6;3(6):386-93. [PubMed: 22783464]

39. Jacquez F, Vaughn LM, Wagner E. Youth as partners, participants or passive recipients: a review of children and adolescents in community-based participatory research (CBPR). Am J Community Psychol. 2013 3;51(1-2):176-89. 10.1007/s10464-012-9533-7 [PubMed: 22718087]

40. De las Nueces D, Hacker K, DiGirolamo A, et al. A systematic review of community-based participatory research to enhance clinical trials in racial and ethnic minority groups. Health Serv Res. 2012 6;47(3 Pt 2):1363-86. Epub 2012 Feb 21. 10.1111/j.1475-6773.2012.01386.x [PubMed: 22353031]

41. Spector AY. CBPR with service providers: arguing a case for engaging practitioners in all phases of research. Health Promot Pract. 2012 3;13(2):252-8. Epub 2011 Jun 15. 10.1177/1524839910382081 [PubMed: 21677113]

42. Stacciarini JM, Shattell MM, Coady M, et al. Review: Community-based participatory research approach to address mental health in minority populations. Community Ment Health J. 2011 10;47(5):489-97. Epub 2010 May 13. 10.1007/s10597-010-9319-z [PubMed: 20464489]

43. Stacciarini JM. A review of community-based participatory research: a promising approach to address depression among Latinos? Issues Ment Health Nurs. 2009 12;30(12):751-7. 10.3109/01612840903177456 [PubMed: 19916809]

44. Cook WK. Integrating research and action: a systematic review of community-based participatory research to address health disparities in environmental and occupational health in the USA. J Epidemiol Community Health. 2008 8;62(8):668-76. 10.1136/jech.2007.067645 [PubMed: 18621950]

45. George S, Duran N, Norris K. A systematic review of barriers and facilitators to minority research participation among African Americans, Latinos, Asian Americans, and Pacific Islanders. Am J Public Health. 2014 2;104(2):e16-31. Epub 2013 Dec 12. 10.2105/AJPH.2013.301706

46. Wallerstein N, Duran B. Community-based participatory research contributions to intervention research: the intersection of science and practice to improve health equity. Am J Public Health. 20104 1;100 Suppl 1:S40-6. Epub 2010 Feb 10. 10.2105/AJPH.2009.184036 [PubMed: 20147663]

47. Robertson LM, Douglas F, Ludbrook A, et al. What works with men? A systematic review of health promoting interventions targeting men. BMC Health Serv Res. 20087 3;8:141 10.1186/1472-6963-8-141 [PubMed: 18598339]

48. Viswanathan M, Ammerman A, Eng E, et al. Community-based participatory research: assessing the evidence. Evid Rep Technol Assess (Summ). 2004 8; (99):1-8. 10.1037/e439622005-001

49. Jagosh J, Macaulay AC, Pluye P, et al. Uncovering the benefits of participatory research: implications of a realist review for health research and practice. Milbank Q. 2012 6;90(2):311-46. 10.1111/j.1468-0009.2012.00665.x [PubMed: 22709390]

50. Jagosh J, Bush PL, Salsberg J, et al. A realist evaluation of community-based participatory research: partnership synergy, trust building and related ripple effects. BMC Public Health. 20157 30;15:725 10.1186/s12889-015-1949-1 [PubMed: 26223523]

51. Moher D, Shamseer L, Clarke M, et al. Preferred reporting items for systematic review and metaanalysis protocols (PRISMA-P) 2015 statement. Syst Rev. 2015 1 1;4:1 10.1186/2046-4053-4-1 [PubMed: 25554246]

52. Shamseer L, Moher D, Clarke M, et al. Preferred reporting items for systematic review and metaanalysis protocols (PRISMA-P) 2015: elaboration and explanation. BMJ. 2015 1 2;350:g7647 10.1136/bmj.g7647 [PubMed: 25555855]

53. Israel BA, Schulz AJ, Parker EA, et al. Review of community-based research: assessing partnership approaches to improve public health. Annu Rev Public Health. 1998;19:173-202. 10.1146/annurev.publhealth.19.1.173 [PubMed: 9611617] 
54. Israel B, Schultz A, Parker E, et al. Critical issues in developing and following CBPR principles In: Minkler M, Wallerstein N, eds. Community-based participatory research for health: from process to outcome. San Francisco, CA: Jossey-Bass, 2008;47-66.

55. Minkler M, Blackwell AG, Thompson M, et al. Community-based participatory research: implications for public health funding. Am J Public Health. 2003 8;93(8):1210-3. 10.2105/ AJPH.93.8.1210 [PubMed: 12893597]

56. McElfish PA, Ayers BL, Purvis RS, et al. Best practices for community-engaged participatory research with Pacific Islander communities in the USA and USAPI: protocol for a scoping review. BMJ Open. 20181 24;8(1):e019653 10.1136/bmjopen-2017-019653

57. Charmaz K. 'Discovering' chronic illness: using grounded theory. Soc Sci Med. 1990;30(11):1161-72. 10.1016/0277-9536(90)90256-R [PubMed: 2360052]

58. Charmaz K. Teaching theory construction with initial grounded theory tools: a reflection on lessons and learning. Qual Health Res. 2015 12;25(12):1610-22. 10.1177/1049732315613982 [PubMed: 26646825]

59. Darling M, Gonzalez F, Graves K, et al. Practical tips for establishing partnerships with academic researchers: a resource guide for community-based organizations. Prog Community Health Partnersh. 2015 Summer;9(2):203-12. 10.1353/cpr.2015.0042 [PubMed: 26412762]

60. Adebayo OW, Salerno JP, Francillon V, et al. A systematic review of components of communitybased organisation engagement. Health Soc Care Community. 2018 7;26(4):e474-84. Epub 2018 Jan 4. 10.1111/hsc.12533 [PubMed: 29314373]

61. Bowers B, Jacobson N, Krupp A. Can lay community advisors improve the clarity of research participant recruitment materials and increase the likelihood of participation? Res Nurs Health. 2017 2;40(1):63-9. Epub 2016 Sep 30. 10.1002/nur.21752 [PubMed: 27686332]

62. Aitaoto N, Campo S, Snetselaar LG, et al. Formative research to inform nutrition interventions in Chuuk and the U.S. Pacific. J Acad Nutr Diet. 2015 6;115(6):947-53. Epub 2015 Jan 27. 10.1016/ j.jand.2014.11.018 [PubMed: 25634092]

63. Aitaoto N, Braun K, Estrella J, et al. Design and results of a culturally tailored cancer outreach project by and for Micronesian women. Prev Chronic Dis. 2012;9:E82 Epub 2012 Apr 5. 10.5888/ pcd9.100262 [PubMed: 22480611]

64. Aitaoto N, Campo SL, Snetselaar LG, et al. Factors inhibiting physical activity as treatment for diabetic chuukese in Chuuk and Hawai'i. Hawaii J Med Public Health. 2017 Sep;76(9):247-52. [PubMed: 28900579]

65. Baumhofer NK, Rothfus N, Yoshimura S, et al. Developing a multicultural nutrition education tool: Pacific Island food models. J Nutr Educ Behav. 2014 Sep-Oct;46(5):451-3. Epub 2014 Mar 15. 10.1016/j.jneb.2014.02.006 [PubMed: 24637064]

66. Braun KL, Tsark J, Santos LA, et al. 'Imi Hale-the Native Hawaiian cancer awareness, research, and training network: second-year status report. Asian Am Pac Isl J Health. 2003 WinterSpring;10(1):4-16. [PubMed: 15352771]

67. Braun KL, Tsark JU, Santos L, et al. Building Native Hawaiian capacity in cancer research and programming. A legacy of 'Imi Hale. Cancer. 200610 15;107(8 Suppl):2082-90. 10.1002/ cncr.22157 [PubMed: 16977599]

68. Bruss MB, Michael TJ, Morris JR, et al. Childhood obesity prevention: an intervention targeting primary caregivers of school children. Obesity (Silver Spring). 2010 1;18(1):99-107. Epub 2009 May 7. 10.1038/oby.2009.111 [PubMed: 19424164]

69. Cook WK, Weir RC, Ro M, et al. Improving Asian American, Native Hawaiian, and Pacific Islander health: national organizations leading community research initiatives. Prog Community Health Partnersh. 2012 Spring;6(1):33-41. [PubMed: 22643786]

70. Delafield R, Hermosura AN, Ing CT, et al. A Community-Based Participatory Research Guided Model for the Dissemination of Evidence-Based Interventions. Prog Community Health Partnersh. 2016;10(4):585-595. 10.1353/cpr.2016.0067 [PubMed: 28569684]

71. DePue JD, Rosen RK, Seiden A, et al. Implementation of a culturally tailored diabetes intervention with community health workers in American Samoa. Diabetes Educ. 2013 Nov-Dec;39(6):761-71. Epub 2013 Sep 19. 10.1177/0145721713504630 [PubMed: 24052204] 
72. DiStefano A, Hui B, Barrera-Ng A, et al. Contextualization of HIV and HPV risk and prevention among Pacific Islander young adults in Southern California. Soc Sci Med. 2012 8;75(4):699-708. Epub 2012 May 8. 10.1016/j.socscimed.2012.04.011 [PubMed: 22647562]

73. Englberger L, Lorens A, Pretrick M, et al. Local food policies can help promote local foods and improve health: a case study from the Federated States of Micronesia. Hawaii Med J. 2011 11;70(11 Suppl 2):31-4. [PubMed: 22235156]

74. Fialkowski MK, DeBaryshe B, Bersamin A, et al. A community engagement process identifies environmental priorities to prevent early childhood obesity: the Children's Healthy Living (CHL) program for remote underserved populations in the U.S. Affiliated Pacific Islands, Hawaii and Alaska. Matern Child Health J. 2014 12;18(10):2261-74. 10.1007/s10995-013-1353-3 [PubMed: 24043557]

75. Gellert K, Braun KL, Morris R, et al. The 'Ohana Day Project: a community approach to increasing cancer screening. Prev Chronic Dis. 2006 7;3(3):A99. [PubMed: 16776900]

76. Hallgren E, McElfish P, Rubon-Chutaro J. Barriers and opportunities: a community-based participatory research study of health beliefs related to diabetes in a U.S. Marshallese community. Diabetes Educ. 2015 2;41(1):86-94. Epub 2014 Nov 14. 10.1177/0145721714559131 [PubMed: 25398722]

77. Helm S, Lee W, Hanakahi V, et al. Using Photovoice with youth to develop a drug prevention program in a rural Hawaiian community. Am Indian Alsk Native Ment Health Res. 2015;22(1):126. 10.5820/aian.2201.2015.1

78. Kagawa-Singer M, Park Tanjasiri S, Lee SW, et al. Breast and cervical cancer control among Pacific Islander and Southeast Asian Women: participatory action research strategies for baseline data collection in California. J Cancer Educ. 2006 Spring;21(1 Suppl):S53-60. 10.1207/ s15430154jce2101s_11 [PubMed: 17020504]

79. Kaholokula JK, Mau MK, Efird JT, et al. A family and community focused lifestyle program prevents weight regain in Pacific Islanders: a pilot randomized controlled trial. Health Educ Behav. 2012 8;39(4):386-95. Epub 2011 May 6. 10.1177/1090198110394174 [PubMed: 21551421]

80. Kaholokula JK, Look M, Mabellos T, et al. Cultural dance program improves hypertension management for Native Hawaiians and Pacific Islanders: a pilot randomized trial. J Racial Ethn Health Disparities. 2017 2;4(1):35-46. Epub 2015 Dec 22. 10.1007/s40615-015-0198-4 [PubMed: 27294768]

81. Kulukulualani M, Braun KL, Tsark JU. Using a participatory four-step protocol to develop culturally targeted cancer education brochures. Health Promot Pract. 2008 10;9(4):344-55. Epub 2008 Mar 19. 10.1177/1524839907302737 [PubMed: 18353907]

82. Kwan PP, Briand G, Lee C, et al. Use of a community-based participatory research approach to assess knowledge, attitudes, and beliefs on biospecimen research among Pacific Islanders. Health Promot Pract. 2014 5;15(3):422-30. Epub 2014 Jan 6. 10.1177/1524839913516464 [PubMed: 24396121]

83. Kwan P, Briand G, Lee C, et al. Reservations to participate in biospecimen research among Pacific Islanders. Calif J Health Promot. 2015 12;13(3):27-33. [PubMed: 29805326]

84. LaBreche M, Cheri A, Custodio H, et al. Let's Move for Pacific Islander communities: an evidence-based intervention to increase physical activity. J Cancer Educ. 2016 6;31(2):261-7. 10.1007/s13187-015-0875-3 [PubMed: 26153489]

85. Leslie JH, Hughes CK, Braun KL. Engaging participants in design of a Native Hawaiian worksite wellness program. Prog Community Health Partnersh. 2010 Summer;4(2):121-30. 10.1353/ cpr.0.0121 [PubMed: 20543487]

86. Magnussen L, Shoultz J, Hansen K, et al. Intimate partner violence: perceptions of Samoan women. J Community Health. 2008 12;33(6):389-94. 10.1007/s10900-008-9110-z [PubMed: 18581217]

87. Magnussen L, Shoultz J, Richardson K, et al. Responding to the needs of culturally diverse women who experience intimate partner violence. Hawaii Med J. 2011 1;70(1):9-15.

88. Mau M, Kaholokula K, West M, et al. Translating diabetes prevention into native Hawaiian and Pacific Islander communities: the PILI 'Ohana Pilot project. Prog Community Health Partnersh. 2010 Spring;4(1):7-16. 10.1353/cpr.0.0111 [PubMed: 20364073] 
89. McElfish PA, Narcisse MR, Long CR, et al. Leveraging community-based participatory research capacity to recruit Pacific Islanders into a genetics study. J Community Genet. 2017 10;8(4):28391. Epub 2017 Jul 8. 10.1007/s12687-017-0313-9 [PubMed: 28689351]

90. McElfish PA, Bridges MD, Hudson JS, et al. Family model of diabetes education with a Pacific Islander community. Diabetes Educ. 2015 12;41(6):706-15. Epub 2015 Sep 11. 10.1177/0145721715606806 [PubMed: 26363041]

91. McElfish P, Moore R, Woodring D, et al. Social ecology and diabetes self-management among Pacific Islanders in Arkansas. J Fam Med Dis Prev. 2016;2(1). pii: 026. Epub 2016 Mar 14. 10.23937/2469-5793/1510026

92. McElfish P, Hallgren E, Henry L, et al. Health beliefs of U.S. Marshallese regarding type 2 diabetes. Am J Health Behav. 2016 3;40(2):248-57. 10.5993/AJHB.40.2.10 [PubMed: 26931757]

93. McElfish PA, Purvis RS, Maskarinec GG, et al. Interpretive policy analysis: Marshallese COFA migrants and the Affordable Care Act. Int J Equity Health. 20166 11;15:91 10.1186/ s12939-016-0381-1 [PubMed: 27286831]

94. McEligot AJ, McMullin J, Pang K, et al. Diet, psychosocial factors related to diet and exercise, and cardiometabolic conditions in Southern Californian Native Hawaiians. Hawaii Med J. 2010 5;69(5 Suppl 2):16-20. [PubMed: 20544604]

95. Mishra SI, Luce PH, Baquet CR. Increasing pap smear utilization among Samoan women: results from a community based participatory randomized trial. J Health Care Poor Underserved. 2009 5;20(2 Suppl):85-101. 10.1353/hpu.0.0160 [PubMed: 19711495]

96. Oneha MF, Magnussen L, Shoultz J. The voices of Native Hawaiian women: perceptions, responses and needs regarding intimate partner violence. Calif J Health Promot. 2010 12;8(1):7281. 10.32398/cjhp.v8i1.2032 [PubMed: 22787436]

97. Oneha MF, Dodgson JE, DeCambra MH, et al. Connecting culturally and spiritually to healthy eating: a community assessment with Native Hawaiians. Asian Pac Isl Nurs J. 2016;1(3):116-26. 10.9741/23736658.1023 [PubMed: 28239642]

98. Scott A, Shreve M, Ayers B, et al. Breast-feeding perceptions, beliefs and experiences of Marshallese migrants: an exploratory study. Public Health Nutr. 2016 11;19(16):3007-16. Epub 2016 May 27. 10.1017/S1368980016001221 [PubMed: 27230629]

99. Shoultz J, Magnussen L, Kreidman N, et al. Engaging Native Hawaiians and Pilipinos in creating supportive and safe violence-free communities for women through a piloted "talkstory" intervention: implications for program development. Eval Program Plann. 2015 8;51:78-84. Epub 2014 Dec 9. 10.1016/j.evalprogplan.2014.12.011 [PubMed: 25541293]

100. Siu A, Palakiko DM. Cancer assessment methodology in a native Hawaiian community. Prog Community Health Partnersh. 2008 Winter;2(4):291-9. 10.1353/cpr.0.0040 [PubMed: 20208309]

101. Tanjasiri SP, Weiss JW, Santos L, et al. CBPR-informed recruitment and retention adaptations in a randomized study of pap testing among Pacific Islanders in Southern California. Prog Community Health Partnersh. 2015 Autumn;9(3):389-96. 10.1353/cpr.2015.0067 [PubMed: 26548790]

102. Tanjasiri SP, Wiersma L, Briand G, et al. Balancing community and university aims in community-based participatory research: a Pacific Islander youth study. Prog Community Health Partnersh. 2011 Spring;5(1):19-25. 10.1353/cpr.2011.0001 [PubMed: 21441665]

103. Tanjasiri SP, Mata'alii S, Hanneman M, et al. Needs and experiences of Samoan breast cancer survivors in Southern California. Hawaii Med J. 2011 11;70(11 Suppl 2): 35-9. [PubMed: 22235157]

104. Braun KL, Mokuau N, Hunt GH, et al. Supports and obstacles to cancer survival for Hawaii's native people. Cancer Pract. 2002 Jul-Aug;10(4):192-200. 10.1046/j.1523-5394.2002.104001.x [PubMed: 12100103]

105. Braun KL, Tsark JU. Community-based research should be based in the community. Prog Community Health Partnersh. 2008 Winter;2(4):271-3. 10.1353/cpr.0.0043 [PubMed: 20208305]

106. Chung-Do J, Ahedo A, Gentry L. Quentin Burdick program: working in rural communities in Hawaii. Prog Community Health Partnersh. 2008 Winter;2(4):341-7. 10.1353/cpr.0.0039 [PubMed: 20208314] 
107. Chung-Do JJ, Look MA, Mabellos T, et al. Engaging Pacific Islanders in research: community recommendations. Prog Community Health Partnersh. 2016 Spring; 10(1):63-71. 10.1353/ cpr.2016.0002 [PubMed: 27018355]

108. DiStefano A, Peters R, Tanjasiri SP, et al. A community-based participatory research study of HIV and HPV vulnerabilities and prevention in two Pacific Islander communities: ethical challenges and solutions. J Empir Res Hum Res Ethics. 2013 2;8(1):68-78. 10.1525/ jer.2013.8.1.68 [PubMed: 23485672]

109. Helm S, Okamoto S. Developing the Ho'ouna Pono substance use prevention curriculum: collaborating with Hawaiian youth and communities. Hawaii J Med Public Health. 2013 2;72(2):66-9. [PubMed: 23463854]

110. McElfish PA, Goulden PA, Bursac Z, et al. Engagement practices that join scientific methods with community wisdom: designing a patient-centered, randomized control trial with a Pacific Islander community. Nurs Inq. 2017 4;24(2). Epub 2016 Jun 20. 10.1111/nin.12141

111. McElfish PA, Kohler P, Smith C, et al. Community-driven research agenda to reduce health disparities. Clin Transl Sci. 2015 12;8(6):690-5. Epub 2015 Nov 17. 10.1111/cts.12350 [PubMed: 26573096]

112. McMullin J, Bone M, Pang J, et al. Native Hawaiian voices: enhancing the role of cultural values in community based participatory research. Calif J Health Promot. 2010 12;8:52-62. 10.32398/ cjhp.v8iSI.2042 [PubMed: 25076863]

113. Mokuau N, Browne CV, Braun KL, et al. Using a community-based participatory approach to create a resource center for Native Hawaiian elders. Educ Health (Abingdon). 2008 12;21(3):174 Epub 2008 Dec 3. [PubMed: 19967637]

114. Moleta CD, Look MA, Trask-Batti MK, et al. 2016 Writing Contest graduate winner: Cardiovascular disease training for community health workers serving Native Hawaiians and other Pacific Peoples. Hawaii J Med Public Health. 2017 7;76(7):190-8. [PubMed: 28721313]

115. Nacapoy AH, Kaholokula JK, West MR, et al. Partnerships to address obesity disparities in Hawaiì: The PILI Òhana project. Hawaii Med J. 2008 9;67(9):237-41. [PubMed: 18853898]

116. Townsend CK, Dillard A, Hosoda KK, et al. Community-based participatory research integrates behavioral and biological research to achieve health equity for Native Hawaiians. Int J Environ Res Public Health. 201512 22;13(1):ijerph13010004. 10.3390/ijerph13010004

117. Tran JH, Mouttapa M, Ichinose TY, et al. Sources of information that promote breast and cervical cancer knowledge and screening among native Hawaiians in Southern California. J Cancer Educ. 2010 12;25(4):588-94. 10.1007/s13187-010-0078-x [PubMed: 20237883]

118. McElfish PA, Long CR, Rowland B, et al. Improving culturally appropriate care using a community-based participatory research approach: evaluation of a multicomponent cultural competency training program, Arkansas, 2015-2016. Prev Chronic Dis. 20178 3;14:E62 10.5888/pcd14.170014 [PubMed: 28771402]

119. McElfish PA, Rowland B, Long CR, et al. Diabetes and hypertension in Marshallese adults: results from faith-based health screenings. J Racial Ethn Health Disparities. 2017 12;4(6):104250. Epub 2016 Nov 11. 10.1007/s40615-016-0308-y [PubMed: 27837454]

120. Oneha MF, Dodgson JE. Refining the research infrastructure at community health centers. Prog Community Health Partnersh. 2014 Spring;8(1):61-5. 10.1353/cpr.2014.0011 [PubMed: 24859103]

121. Sy A, Hernandez B, Tareg A, et al. Acceptability and feasibility of a community based participatory research project comparing cytology and urine HPV DNA testing for cervical cancer screening in Yap, Federated States of Micronesia. Cancer Epidemiol. 2017 10;50(Pt B):283-8. 10.1016/j.canep.2017.07.008 [PubMed: 29120838]

122. Ta V, Chao J, Kaholokula J. Cultural Identity and Conceptualization of Depression among Native Hawaiian Women. AAPI Nexus. 2010 Fall;8(2):63-86. 10.17953/appc.8.2.5533612679732611

123. Sinclair KA, Makahi EK, Shea-Solatorio C, et al. Outcomes from a diabetes self-management intervention for Native Hawaiians and Pacific People: partners in care. Ann Behav Med. 2013 2;45(1):24-32. 10.1007/s12160-012-9422-1 [PubMed: 23086589] 
124. Helm S, Okamoto SK, Medeiros H, et al. Participatory drug prevention research in rural Hawai'i with native Hawaiian middle school students. Prog Community Health Partnersh. 2008 Winter;2(4):307-13. 10.1353/cpr.0.0042 [PubMed: 19696900]

125. Paige C, Peters R, Parkhurst M, et al. Enhancing community-based participatory research partnerships through appreciative inquiry. Prog Community Health Partnersh. 2015 Autumn;9(3):457-63. 10.1353/cpr.2015.0054 [PubMed: 26548798]

126. Purvis R, Bing WI, Jacob CJ, et al. Community health warriors: Marshallese community health workers' perceptions and experiences with CBPR and community engagement. Prog Community Health Partnersh. 2017;11(3):315-20. 10.1353/cpr.2017.0037 [PubMed: 29056624]

127. Kwan P, Sabado-Liwag M, Lee C, et al. Development of an online smoking cessation curriculum for Pacific Islanders: a community-based participatory research approach. Prog Community Health Partnersh. 2017;11(3):263-74. 10.1353/cpr.2017.0031 [PubMed: 29056618]

128. Panapasa S, Jackson J, Caldwell C, et al. Community-based participatory research approach to evidence-based research: lessons from the Pacific Islander American health study. Prog Community Health Partnersh. 2012 Spring;6(1):53-8. 10.1353/cpr.2012.0013 [PubMed: 22643788]

129. Santos L, Braun KL, Ae'a K, et al. Institutionalizing a comprehensive tobacco-cessation protocol in an indigenous health system: lessons learned. Prog Community Health Partnersh. 2008 Winter;2(4):279-89. 10.1353/cpr.0.0038 [PubMed: 20208308]

130. Tanjasiri SP, Tran JH, Palmer PH, et al. Developing a community-based collaboration to reduce cancer health disparities among Pacific Islanders in California. Pac Health Dialog. 2007 3;14(1):119-27.

131. Tisnado DM, Sablan-Santos L, Guevara L, et al. A case study in Chamorro community and academic engagement for a community-partnered research approach. Calif J Health Promot. 2010 12;8(SE):39-51. 10.32398/cjhp.v8iSI.2041 [PubMed: 26726298]

132. Tsark JA. A participatory research approach to address data needs in tobacco use among Native Hawaiians. Asian Am Pac Isl J Health. 2001 Winter-Spring;9(1):40-8. [PubMed: 11720413]

133. Walsh MS, Hope E, Isaia L, et al. Prevalence of Chlamydia trachomatis infection in Samoan women aged 18 to 29 and assessment of possible risk factors: a community-based study. Trans $\mathrm{R}$ Soc Trop Med Hyg. 2015 4;109(4):245-51. Epub 2015 Mar 1. 10.1093/trstmh/trv014 [PubMed: 25732755]

134. Yeary KH, Aitaoto N, Sparks K, et al. Cultural adaptation of diabetes self-management education for marshallese residing in the United States: lessons learned in curriculum development. Prog Community Health Partnersh. 2017;11(3):253-61. 10.1353/cpr.2017.0030 [PubMed: 29056617]

135. Webster J, Snowdon W, Moodie M, et al. Cost-effectiveness of reducing salt intake in the Pacific Islands: protocol for a before and after intervention study. BMC Public Health. 2014 2 4;14:107 10.1186/1471-2458-14-107 [PubMed: 24495646]

136. Tsark J', Braun KL. Na Liko Noelo: a program to develop Native Hawaiian researchers. Pac Health Dialog. 2004 Sep;11(2):225-32. [PubMed: 16281705]

137. Kagawa-Singer M, Pourat N, Breen N, et al. Breast and cervical cancer screening rates of subgroups of Asian American women in California. Med Care Res Rev. 2007 12;64(6):706-30. Epub 2007 Sep 5. 10.1177/1077558707304638 [PubMed: 17804823]

138. Yeary K, Aitaoto N, Sparks K, et al. Cultural adaptation of diabetes self-management education for Marshallese residing in the U.S.: lessons learned in curriculum development. Prog Community Health Partnersh. 2017;11(3):253-61. 10.1353/cpr.2017.0030 [PubMed: 29056617]

139. Yeary K, Cornell C, Prewitt E, et al. The WORD (Wholeness, Oneness, Righteousness, Deliverance): design of a randomized controlled trial testing the effectiveness of an evidencebased weight loss and maintenance intervention translated for a faith-based, rural, African American population using a community-based participatory approach. Contemp Clin Trials. 2015 1;40:63-73. Epub 2014 Nov 15. 10.1016/j.cct.2014.11.009 [PubMed: 25461496]

140. Katz DL, Murimi M, Gonzalez A, et al. From controlled trial to community adoption: the multisite translational community trial. Am J Public Health. 2011 8;101(8): e17-27. Epub 2011 Jun 16. 10.2105/AJPH.2010.300104 [PubMed: 21680935] 
141. McElfish P, Post J, Rowland B. A social ecological and community-engaged perspective for addressing health disparities among Marshallese in Arkansas. Int J Nurs Clin Pract. 2016;3(191):1-6. 10.15344/2394-4978/2016/191

142. Niide TK, Davis J, Tse AM, et al. Evaluating the impact of a school-based prevention program on self-esteem, body image, and risky dieting attitudes and behaviors among Kaua'i youth. Hawaii J Med Public Health. 2013 8;72(8):273-8. [PubMed: 24349890]

143. Townsend C. Impacts of Hawaiian language loss and promotion via the linguistic landscape [dissertation]. Manoa, Hawaii: University of Hawaii at Manoa, 2014 Available at: https:// scholarspace.manoa.hawaii.edu/bitstream/10125/100360/Townsend_Claire_r.pdf.

144. Massey PD, Wakageni J, Kekeubata E, et al. TB questions, East Kwaio answers: communitybased participatory research in a remote area of Solomon Islands. Rural Remote Health. 2012;12:2139 Epub 2012 Oct 24. [PubMed: 23094978]

145. Vawer M, Kaina P, Leonard A, et al. Navigating the cultural geography of indigenous peoples' attitude toward genetic research: the Ohana (family) heart project. Int J Circumpolar Health. 2013 8 5;72 10.3402/ijch.v72i0.21346

146. Israel BA, Eng E, Schulz AJ, et al. (eds). Methods for community-based participatory research for health. San Francisco, CA: Jossey-Bass, 2012.

147. Kaholokula JK, Ing CT, Look MA, et al. Culturally responsive approaches to health promotion for Native Hawaiians and Pacific Islanders. Ann Hum Biol. 2018 5;45(3): 249-63. Epub 2018 May 29. 10.1080/03014460.2018.1465593 [PubMed: 29843522]

148. Office of Management Assessment. 11601-Food and Beverage; Entertainment. Rockville, MD: National Institutes of Health, 2016 Available at: https://policymanual.nih.gov/1160-1.

149. Goins RT, Garroutte EM, Fox SL, et al. Theory and practice in participatory research: lessons from the Native Elder Care Study. Gerontologist. 2011 6;51(3):285-94. Epub 2011 Feb 3. 10.1093/geront/gnq130 [PubMed: 21292753]

150. Rasmus SM. Indigenizing CBPR: evaluation of a community-based and participatory research process implementation of the Elluam Tungiinun (towards wellness) program in Alaska. Am J Community Psychol. 2014 9;54(1-2):170-9. 10.1007/s10464-014-9653-3 [PubMed: 24756887]

151. Hodge FS, Pasqua A, Marquez CA, et al. Utilizing traditional storytelling to promote wellness in American Indian communities. J Transcult Nurs. 2002 1;13(1):6-11. 10.1177/104365960201300102 [PubMed: 11776018]

152. Okihiro M, Harrigan R. An overview of obesity and diabetes in the diverse populations of the Pacific. Ethn Dis. 2005 Autumn;15(4 Suppl 5):S5-71-80.

153. Look M, Trask-Batti M, Agres R, et al. Assessment and priorities for health \& well-being in Native Hawaiians and other Pacific peoples. Honolulu, HI: Center for Native and Pacific Health Disparities Research, University of Hawaii, 2013 Available at: http://www2.jabsom.hawaii.edu/ native/docs/community/DNHH_Asssessment\&Priorities_Report_2013.pdf.

154. Bharadwaj L. A framework for building research partnerships with First Nations communities. Environ Health Insights. 2014 8;8:15-25. 10.4137/EHI.S10869

155. Pahwa P, Abonyi S, Karunanayake C, et al. A community-based participatory research methodology to address, redress, and reassess disparities in respiratory health among First Nations. BMC Res Notes. 20155 16;8:199 10.1186/s13104-015-1137-5 [PubMed: 25981585]

156. Kyoon-Achan G, Lavoie J, Avery Kinew K, et al. Innovating for transformation in First Nations health using community-based participatory research. Qual Health Res. 2018 6;28(7):1036-49. Epub 2018 Feb 27. 10.1177/1049732318756056 [PubMed: 29484964]

157. Tobias JK, Richmond CAM, Luginaah I. Community-based participatory research (CBPR) with Indigenous communities: producing respectful and reciprocal research. J Empir Res Hum Res Ethics. 2013 4;8(2):129-40. 10.1525/jer.2013.8.2.129 [PubMed: 23651937] 


\section{Box 1.}

\section{ELIGIBILITY CRITERIA}

Participant

population:

Study type:

Outcomes:

Context:

Study methods:
CBPR studies conducted with Pacific Islanders in the US or USAPI.

Studies that aggregate Pacific Islander and Asian American data were excluded.

Studies conducted with Pacific Islanders outside the US or USAPI were not included.

All types of studies focused on physical health and/or mental health.

Specific CBPR best practices in research conducted with Pacific Islanders.

Articles that self-identify as using a CBPR approach (or other engaged research term such as action research or patient-centered research).

All types of studies (e.g., randomized controlled trials, mixed methods, cross sectional, descriptive, qualitative, case studies, etc.). 


\section{Box 2}

\section{DATA ITEMS}

Participant population

- $\quad$ Race/ethnicity including subgroup of Pacific Islander

- Geographic location

- $\operatorname{Sex}$

- $\quad$ Age group

- Health issue of focus

Study method(s)

- Qualitative

- Quantitative

- $\quad$ Mixed methods

- Descriptive

Best Practices Publication details

- $\quad$ Best practices related to CBPR

- Authors

- $\quad$ Article citation

- $\quad$ Funding source 


\section{Best Practices for Community-Engaged Research}

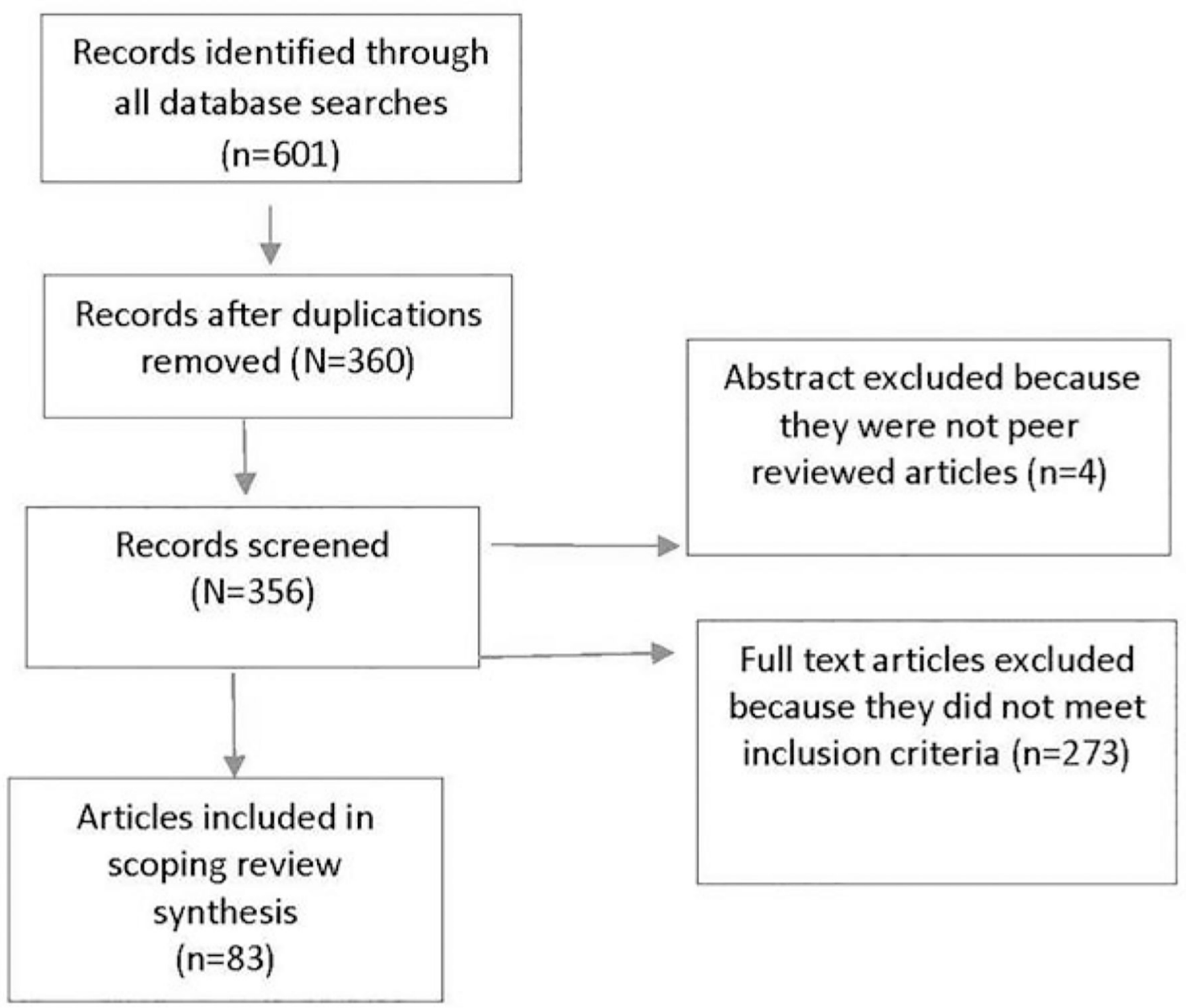

Figure 1.

PRISMA flow diagram of selected articles. 
Table 1.

\section{METHODS}

\begin{tabular}{lcc} 
Methods & N & Percent $^{\boldsymbol{a}}$ \\
\hline Qualitative only & 27 & $33 \%$ \\
Mixed Methods & 27 & $33 \%$ \\
Quantitative only & 15 & $18 \%$ \\
Descriptive & 14 & $17 \%$ \\
\hline
\end{tabular}

Note:

${ }^{a}$ Percentages may not total 100 due to rounding 
Table 2.

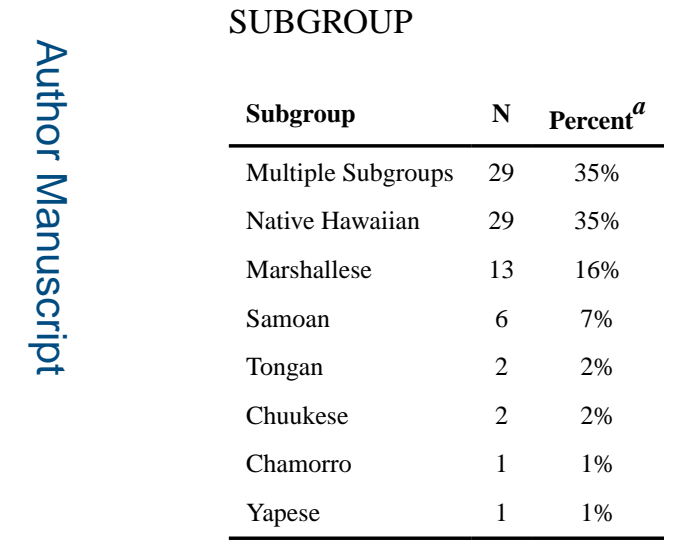

Note:

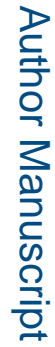

${ }^{a}$ Percentages may not total 100 due to rounding. 
Table 3.

\section{GEOGRAPHIC LOCATION}

\begin{tabular}{lcc} 
Geographic Location & N & Percent $^{\boldsymbol{a}}$ \\
\hline Hawaii only & 41 & $49 \%$ \\
US & 34 & $41 \%$ \\
Multiple Locations in US and USAPI & 3 & $3 \%$ \\
USAPI & 5 & $6 \%$ \\
\hline Note: & & \\
a Percentages may not total 100 due to rounding.
\end{tabular}


Table 4.

\section{GENDER AND AGE}

\begin{tabular}{lcc} 
Gender and Age & N & Percent $^{\boldsymbol{a}}$ \\
\hline Men and Women & 42 & $51 \%$ \\
Women & 16 & $19 \%$ \\
Men & 4 & $5 \%$ \\
Non Specified & 21 & $25 \%$ \\
Adults Only & 56 & $67 \%$ \\
Children Only & 5 & $6 \%$ \\
Adults and Children & 5 & $6 \%$ \\
Non Specified & 17 & $20 \%$ \\
\hline
\end{tabular}

Note:

${ }^{a}$ Percentages may not total 100 due to rounding. 
Table 5.

\section{HEALTH DISPARITIES}

\begin{tabular}{lcc} 
Health Disparities & N & Percent $^{\boldsymbol{a}}$ \\
\hline Cancer & 16 & $19 \%$ \\
Diabetes & 15 & $18 \%$ \\
Non-specific Health Research & 14 & $16 \%$ \\
Obesity & 12 & $14 \%$ \\
Drug Prevention & 7 & $8 \%$ \\
Intimate Partner Violence & 4 & $5 \%$ \\
Cardiovascular Disease & 4 & $5 \%$ \\
Maternal Health & 3 & $4 \%$ \\
Sexually Transmitted Disease & 3 & $4 \%$ \\
Biospecimen Research & 2 & $2 \%$ \\
Genetic Studies & 2 & $2 \%$ \\
Depression & 1 & $1 \%$ \\
\hline
\end{tabular}

Note:

a Percentages may not total 100 due to rounding. 
Table 6.

\section{BEST PRACTICES THEMES AND SUB-THEMES}

\begin{tabular}{llcc} 
Best Practices Themes & \multicolumn{1}{c}{ Sub-Themes } & Percent & N \\
\hline Engaging Community Leaders & Pacific Islander Research Staff & $81 \%$ & 67 \\
& Community-Based Organizations & $80 \%$ & 66 \\
& Community Advisory Board & $54 \%$ & 45 \\
& Faith-Based Leader/Elders & $22 \%$ & 18 \\
Facilitators and Barriers to Research & Location & $42 \%$ & 35 \\
& Language & $36 \%$ & 30 \\
& Time for Relationships & $23 \%$ & 19 \\
& Faith/God & $23 \%$ & 19 \\
& Oral Traditions and Talk Story & $19 \%$ & 16 \\
& Sharing a Meal & $17 \%$ & 14 \\
& Separate Engagement Based Upon Sex & $7 \%$ & 6 \\
Honoring Cultural Practices & Group Engagement & $55 \%$ & 46 \\
& Family Engagement & $39 \%$ & 32 \\
& Collect Consent & $24 \%$ & 20 \\
\hline
\end{tabular}

J Health Care Poor Underserved. Author manuscript; available in PMC 2020 March 11. 